\title{
SUMS OF INCOMPRESSIBLE SURFACES
}

\author{
ULRICH OERTEL
}

(Communicated by Haynes R. Miller)

\begin{abstract}
In an orientable Haken 3-manifold, given two orientable incompressible surfaces $F$ and $S$ transverse to each other with intersections suitably simplified, certain cut-and-paste operations along curves of intersection yield embedded incompressible surfaces. We show in this paper that, no matter how $F$ and $S$ are isotoped, as long as intersections are suitably simplified, exactly the same finite (possibly empty) set of isotopy classes of incompressible surfaces result from cut-and-paste operations.
\end{abstract}

0. Introduction and statement of results. Throughout this paper we let $M$ be an irreducible, $\partial$-irreducible, orientable 3-manifold. Given two incompressible surfaces $F$ and $S$, transverse to each other and with simplified intersection, it is possible to cut $F$ and $S$ on curves of intersection and reglue (in different ways) to obtain a properly embedded surface, $G$ say. This operation is traditionally called a cut-and-paste operation. Often, depending on choices, $G$ will not be incompressible. We study in this paper those cut-and-paste operations which do yield incompressible surfaces. We address the following:

QUESTION 0. Suppose $F$ and $S$ are orientable incompressible surfaces intersecting transversly. Suppose $F^{\prime}$ and $S^{\prime}$ are isotopic to $F$ and $S$ respectively. Finally, suppose $F^{\prime}$ and $S^{\prime}$ also intersect transversely. If an incompressible surface $G$ is obtained from $F$ and $S$ by a cut-and-paste operation, can a surface isotopic to $G$ be obtained from $F^{\prime}$ and $S^{\prime}$ by cut-and-paste?

We shall see that we cannot hope for a positive answer to the question without imposing conditions on the intersection of $F$ and $S\left(F^{\prime}\right.$ and $\left.S^{\prime}\right)$. Suppose $F, S$ are orientable incompressible, $\partial$-incompressible surfaces in $M$. If $F \cap S$ contains contractible closed curves, we can eliminate them by isotopy of $F$ or $S$. Similarly, if $F \cap S$ contains arcs which are isotopic in $F$ or $S$ to an arc in $\partial F$ or $\partial S$, these can be eliminated by isotopy. For simplicity, we call these arcs contractible curves also. If $\alpha$ is an arc, we use $R=(\alpha \times I) / \sim$ to denote a product with $x \times I$ collapsed to a point for each $x \in \partial \alpha$. Given such a product embedded in $\partial M$ with $\alpha \times 1 \subset \partial F, \alpha \times 0 \subset \partial S$, and $R \cap(S \cup F)=\partial R$, we can isotope $(F, \partial F)$ or $(S, \partial S)$ in $(M, \partial M)$ to eliminate two intersections of $\partial F$ and $\partial S$. Similarly, if $W$ is a compact surface with boundary and $\gamma$ is a closed 1-submanifold of $\partial W$, we use

Received by the editors September 8, 1986 and, in revised form, November 10, 1986. This paper was presented at an AMS meeting in Los Angeles, November 14, 1987.

1980 Mathematics Subject Classification (1985 Revision). Primary 57M99; Secondary 57R52.

Key words and phrases. Incompressible surface, incompressible branched surface, Haken manifold, projective lamination space.

This research was supported by National Science Foundation Grants DMS-8601460, DMS8610730(1), and DMS-8120790. 


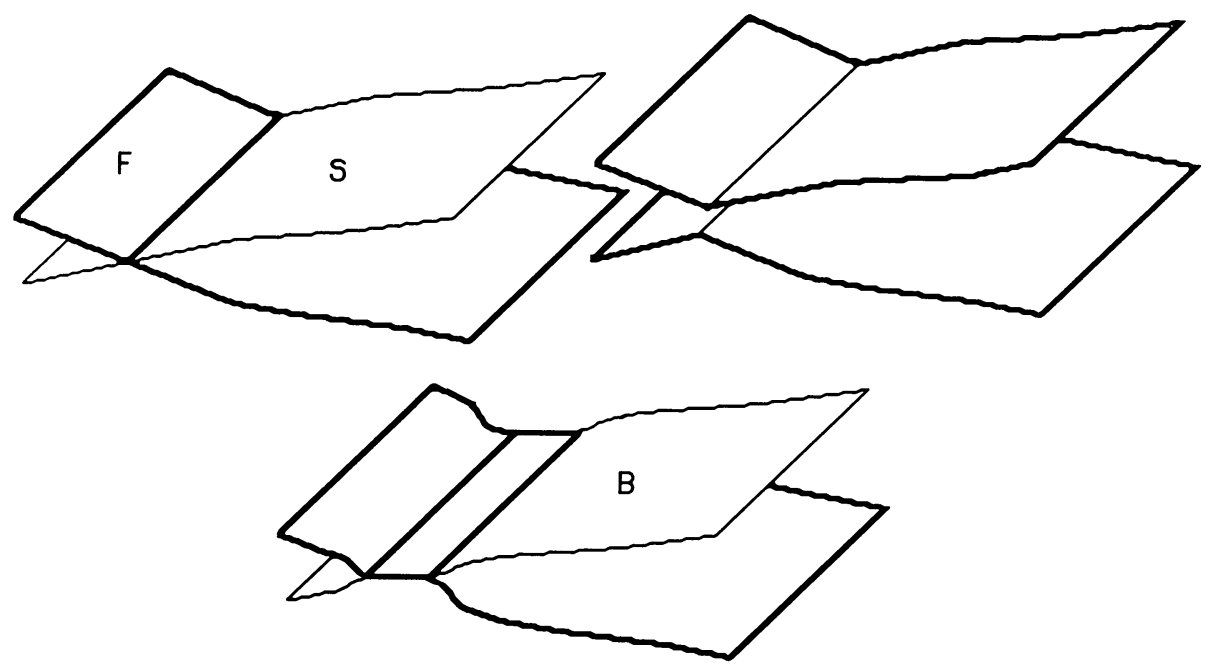

FIGURE 0.1

$P=(W \times I) / \sim$ to denote the product with intervals $x \times I$ collapsed to points for $x \in \gamma$. If $(P,(\partial W-\stackrel{\circ}{\gamma}) \times I)$ is embedded in $(M, \partial M)$ with $W \times 0 \subset S, W \times 1 \subset F$, and $P \cap(S \cup F)=\partial P-\operatorname{int}(P \cap \partial M)$, then we can use the product $P$ to eliminate some curves of $F \cap S$ by isotoping $W \times 1 \subset F$ to $W \times 0 \subset S$.

Suppose we remove all contractible curves of $F \cap S$ by isotopy of $F$ or $S$, then we use products in $\partial M$ between $\operatorname{arcs}$ of $\partial F$ and $\partial S$ to eliminate intersections of $\partial F$ and $\partial S$ until no further such products exist. Suppose, finally, that we remove curves of $F \cap S$ using products in $M$ until no further such products remain. Then we say the intersection $F \cap S$ is simplified.

If $F \cap S$ or $F^{\prime} \cap S^{\prime}$ is not simplified, the Question 0 has a negative answer, as one can easily see using examples in manifolds of the form $M=($ surface $) \times S^{1}$, with surfaces $F$ and $S$ of the form (essential curve) $\times S^{1}$.

Given two incompressible surfaces $F$ and $S$ as above, transverse to each other and with simplified intersection, we consider the possible cut-and-paste operations. On each curve of $F \cap S$ there are two choices for the reglueing, one of which is shown in Figure 0.1. The choice of the reglueing along the curve can be specified by "pinching" $F \cup S$ along curves of intersection to give $F \cup S$ a branched surface structure as indicated in Figure 0.1. We isotope the surfaces near the curve so that they are everywhere tangent along the curve, and so the curve is replaced by a "band of contact." If we choose such a pinching along each curve of $F \cap S$, we obtain a branched surface $F \cup S=B$. If the branched surface $B$ is incompressible, then the theory of incompressible branched surfaces (see [FO, O1, or O2]) shows that the corresponding cut-and-paste operation yields a surface $G$ which is fully carried by $B$ (carried with positive weights by $B$ ), and hence is incompressible. If the surface $G$ is nonorientable, then the incompressibility of $B$ also implies that $G$ is injective, which means that $\partial N(G)$ is incompressible. Because the cut-and-paste operation can be interpreted as the addition of invariant measures on $B$, we say that $G$ is the sum of $F$ and $S$ in $B$, but only when $B$ is incompressible. 
With $F$ and $S$ in a fixed position with simplified intersection, clearly there are just finitely many branched-surface structures on $F \cup S$. Hence there just finitely many branched-surface structures $B=F \cup S$ with $B$ incompressible. Therefore there are just finitely many possible sums $F+S$. The following theorem provides an answer to Question 0, for cut-and-paste operations in incompressible branched surfaces.

THEOREM 1. Let $M$ be an orientable, irreducible, $\partial$-irreducible 3-manifold. Suppose $F_{0}$ and $S_{0}$ are orientable incompressible, $\partial$-incompressible surfaces in $M$ with simplified intersection, and suppose $F_{1}$ and $S_{1}$ are isotopic to $F_{0}$ and $S_{0}$ respectively, also with simplified intersection. Suppose there is a branched-surface structure $B_{0}$ for $F_{0} \cup S_{0}$ such that $B_{0}$ is incompressible. Then there is a corresponding incompressible-branched-surface structure $B_{1}$ for $F_{1} \cup S_{1}$ such that the sum $F_{0}+S_{0}$ in $B_{0}$ is isotopic to the sum $F_{1}+S_{1}$ in $B_{1}$.

Let $S$ denote the set of isotopy classes of orientable, incompressible, $\partial$-incompressible surfaces in $M$. Let $S^{\prime}$ be the set of injective, $\partial$-injective surfaces in $M$, so $S^{\prime}$ is $S$ union the set of nonorientable injective, $\partial$-injective surfaces. With the aim of defining something like an addition operation on $S$, we define a function $p: S \times$ $S \rightarrow$ (finite subsets of $S^{\prime}$ ) as follows. Given a pair of isotopy classes of incompressible surfaces, we choose representatives $F$ and $S$ with simplified intersection. Then $p([F],[S])$ is defined to be the set of isotopy classes of surfaces obtained from $F \cup$ $S$ by cut-and-paste operations corresponding to incompressible-branched-surface structures for $F \cup S$.

COROllaRY 2. The function $p: S \times S \rightarrow\left(\right.$ finite subsets of $\left.S^{\prime}\right)$ is well defined.

The corollary is an immediate consequence of the theorem.

We examine next a less restrictive class of cut-and-paste operations, namely the class of cut-and-pastes involving pairs of orientable incompressible surfaces with simplified intersection and yielding injective surfaces. In this context, we can also answer Question 0.

THEOREM 3. Let $M$ be an orientable, irreducible, $\partial$-irreducible 3-manifold. Suppose $F_{0}$ and $S_{0}$ are orientable incompressible, $\partial$-incompressible surfaces in $M$ with simplified intersection, and suppose $F_{1}$ and $S_{1}$ are isotopic to $F_{0}$ and $S_{0}$ respectively, also with simplified intersection. Suppose a cut-and-paste operation applied to $F_{0}$ and $S_{0}$ yields an injective, $\partial$-injective surface $G_{0}$. Then there is a corresponding cut-and-paste of $F_{1}$ and $S_{1}$ yielding a surface $G_{1}$ isotopic to $G_{0}$.

We define a function $q: S \times S \rightarrow$ (finite subsets of $S^{\prime}$ ) as follows. Given a pair of isotopy classes of incompressible surfaces, we choose representatives $F$ and $S$ with simplified intersection. Then $q([F],[S])$ is defined to be the set of isotopy classes of injective surfaces obtained from $F \cup S$ by cut-and-paste operations. Theorem 3 immediately proves the following:

COROLlaRY 4. The function $q: S \times S \rightarrow\left(\right.$ finite subsets of $\left.S^{\prime}\right)$ is well defined.

It is also clear that given $F, S$ incompressible, $p([F],[S]) \subset q([F],[S])$. The inclusion is sometimes strict, as can be seen using examples in (surface) $\times S^{1}$.

Theorem 1 and Corollary 2 show that there is a finite collection of incompressible "sums" of two given incompressible surfaces. This fact, while interesting in itself, 
is also intimately connected to the structure of the projective lamination space of the 3-manifold $M$. Indeed, I plan to use the ideas in this paper for further study of the projective lamination spaces of 3-mainfolds. For the beginnings of a description of the projective lamination space of a 3-mainfold see [O2 and FH]. Allen Hatcher is currently working on another paper on the subject.

Some of the ideas in this paper have origins in Haken's normal surface theory (see $[\mathbf{H}]$ ). There is a technical fact about normal surfaces, to be proved in another paper, which follows from Theorem 1. Namely, if an open face of the projective solution space (see [JO]) contains a point corresponding to a minimal complexity incompressible surface, then a surface corresponding to any other point in the closed face also has minimal complexity.

The cut-and-paste operations in Theorem 3 and Corollary 4, which yield incompressible surfaces but do not necessarily take place in incompressible branched surfaces, are less natural because they are not important in normal surface theory and have no significance for the projective lamination spaces of 3-manifolds.

I am grateful for support provided by the Mathematical Sciences Research Institute and by the Institute for Advanced Study. I thank Allen Hatcher and Peter Scott for their help.

1. One-parameter simplification of intersections. For simplicity, we shall give all proofs only in the case $\partial M=\varnothing$.

We begin by analyzing in greater detail the products which are used to complete the simplification of intersection of two transverse incompressible surfaces $F$ and $S$, once all contractible curves of intersection have already been eliminated. Suppose there is an embedded product $P=W \times I / \sim$ in $M$ with $W \times 0 \subset S$ and $W \times 1 \subset F$, but suppose that $F$ and $S$ are not necessarily disjoint from $\stackrel{\circ}{P}$. The following lemma shows that the existence of such a product implies the existence of another such product $P$, but with $P \cap(F \cup S)=\partial P$.

LEMMA 1.1. Suppose $G$ is an incompressible surface in the product $W \times I$, and suppose $G \cap(\partial W \times I)=\varnothing$. Then $G$ can be isotoped so $G$ is in Morse position with no centers except on components of $G$ which cobound products with subsurfaces of $W \times 0$ or $W \times 1$. The saddles can be assumed to occur at distinct levels.

The lemma is a special case of a more general result about incompressible surfaces in foliated manifolds. (To prove Theorems 1 and 3 in the case $\partial M \neq \varnothing$, one needs a version of the above lemma in which the vertical boundary $\partial G \cap \partial W \times I$ can contain some arcs essential in $\partial W \times I$.)

PROPOSITION 1.2. Let $S$ and $F$ be orientable, incompressible, $\partial$-incompressible surfaces in $M$. Suppose $F_{t}(0 \leq t \leq 1)$ is an isotopy from $F=F_{0}$ to $F_{1}$ and suppose that for $t=0,1$ the intersection of $F_{t}$ and $S$ is transverse and simplified. Then the isotopy $F_{t}$ can be replaced by an isotopy $F_{t}$ from $F_{0}$ to $F_{1}$ with the intersection of $F_{t}$ and $S$ transverse and simplified except at a finite number of isolated times. At these isolated times an event of the type shown in Figure 2.1 occurs.

PROOF. (Some of the methods and notation in this proof are taken from papers of Allen Hatcher [H1, H2]. In his papers, however, the goal is to eliminate all intersections between a pair of surfaces.) 


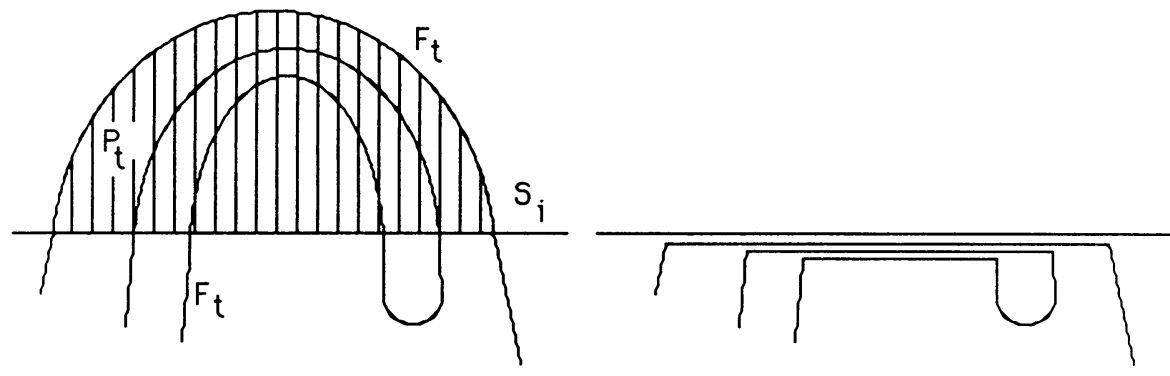

FIGURE 1.3

Choose a bicollar neighborhood $N(S)=S \times[-1,1]$ of the surface $S$. By Sard's Theorem, we may choose finitely many distinct slices $S \times r_{i}=S_{i}$ in $N(S)$ and corresponding intervals $B_{i} \subset I=[0,1]$ such that $F_{t}$ is transverse to $S_{i}$ for each $t \in B_{i}$ and such that the interiors (relative to $I$ ) of the $B_{i}$ 's cover $I$. We suppose the intervals $B_{i}$ are ordered $B_{1}, \ldots, B_{n}$ in an order consistent with the order relation on $I$. So $0 \in B_{1}, 1 \in B_{n}$, and if $B_{i}=\left[c_{i}, d_{i}\right]$, then $c_{i}<d_{i-1}<c_{i+1}<d_{i}$. Further we may assume that $F_{t} \cap S_{1}\left(F_{t} \cap S_{n}\right)$ is simplified for $t \in B_{1}\left(t \in B_{n}\right)$.

Our first goal is to eliminate, for all $i$, all contractible curves from $F_{t} \cap S_{i}$ when $t \in B_{i}$. Given $t \in B_{i}$ let $C_{t}^{i}$ be the collection of contractible curves of $F_{t} \cap S_{i}$. For the same fixed $t$, let $C_{t}=\bigcup_{i} C_{t}^{i}$, where the union is over all $i$ (one or two $i$ ) such that $t \in B_{i}$. Each closed curve $c_{t} \in C_{t}^{i}$ bounds a unique disc $D_{F}\left(c_{t}\right)$ in $F_{t}$, and a unique disc $D_{S}\left(c_{t}\right)$ in $S_{i}$.

If for some $t$ and some $c_{t} \in C_{t}^{i}, \stackrel{\circ}{D}_{F}\left(c_{t}\right)$ contains no other curves of $C_{t}$, so that $c_{t}$ is innermost on $F_{t}$, then $D_{F}\left(c_{t}\right) \cup D_{S}\left(c_{t}\right)$ bounds an embedded ball $P_{t}$, which can be given a product structure $P_{t}=W_{t} \times I / \sim$. The product $P_{t}$ defines an isotopy of $F_{t}$, as shown in Figure 1.3, eliminating $c_{t}$, and possible some other curves of intersection from $C_{t}^{i}$.

Each $c_{t}$ belongs to a continuous 1-parameter family $c_{t}, t \in B_{i}$, for some $i$. We seek a family $c_{t}$ such that $c_{t}$ is innermost in $F_{t}$ for all $t \in B_{i}$. That is, we seek a family $c_{t}$ such that for $t \in B_{i-1} \cap B_{i}, D_{F}\left(c_{t}\right) \cap S_{i-1}=\varnothing$ and for $t \in B_{i} \cap B_{i+1}$, $D_{F}\left(c_{t}\right) \cap S_{i+1}=\varnothing$. If for some $t, C_{t} \neq \varnothing$, such a family $c_{t}$ is easy to find, as follows. Choose some $r$ such that for $t \in B_{r-1} \cap B_{r}, C_{t} \neq \varnothing$. Choose a $c_{t} \in C_{t}$ innermost on $F_{t}$ for $t \in B_{r-1} \cap B_{r}$, and suppose without loss of generality that $c_{t} \in C_{t}^{r}$. (If $c_{t} \notin C_{t}^{r}$, then $c_{t} \in C_{t}^{r-1}$ and an argument similar to the following applies.) We will refer to this $c_{t}$ as $c_{t}^{r}$ to distinguish it; it belongs to a family $c_{t}^{r}, t \in B_{r}$. If $c_{t}^{r}$ is innermost for $t \in B_{r} \cap B_{r+1}$, we are done. Otherwise $D_{F}\left(c_{t}^{r}\right)$ intersects $S_{r+1}$ for $t \in B_{r} \cap B_{r+1}$, and we find in $\dot{D}_{F}\left(c_{t}^{r}\right)$ a curve $c_{t}^{r+1} \in C_{t}^{r+1}$ innermost on $F_{t}$ for $t \in B_{r} \cap B_{r+1}$. Repeating, we get a sequence $c_{t}^{j} \in C_{t}^{j}, j=r, r+1, r+2, \ldots$. The sequence must end since $C_{t}^{n}=\varnothing$. The last $c_{t}^{j}$ in the sequence is one of the type we have been seeking.

Suppose, then, that we have a family $c_{t}, t \in B_{i}$, such that $c_{t}$ is innermost on $F_{t}$ among curves of $C_{t}$ for all $t \in B_{i}$. There is a corresponding family $P_{t}, t \in B_{i}$, of balls bounded by $D_{F}\left(c_{t}\right) \cup D_{S}\left(c_{t}\right)$. For each $t \in B_{i}$ we define an isotopy $F_{t u}, 0 \leq u \leq 1$, as shown in Figure 1.3, to eliminate $c_{t}$ by isotoping $D_{F}\left(c_{t}\right)$ to $D_{S}\left(c_{t}\right)$ and slightly beyond. Clearly these isotopies can be made into a continuous family of isotopies $F_{t u}, 0 \leq u \leq 1, t \in B_{i}$. We wish to make it a continuous family for $t \in I$. To do 
this choose an interval $B_{i}^{\prime}$ in the interior of $B_{i}$ such that the interiors of the $B_{i}$ 's still cover $I$ when $B_{i}$ is replaced by $B_{i}^{\prime}$. Let $Z$ be a quadrilateral disc in $B_{i} \times[0,1]$ with base $B_{i} \times 0$ and opposite side $B_{i}^{\prime} \times 1$. The quadrilateral $Z$, contained in the $(t, u)$ parameter space $[0,1] \times[0,1]$, has sides which taper inward with increasing $u$. Now redefine $F_{t u}$ so that for each fixed $t \in I$, the isotopy continues only as long as $(t, u) \in Z$. If $t \notin B_{i}$ then $F_{t u}$ is the constant isotopy. This family $F_{t u}$ is a continuous family of isotopies.

If $B_{i}$ is replaced by $B_{i}^{\prime}$ and $F_{t}$ is replaced by $F_{t 1}$, then the new $F_{t}$ has the same properties as the old, but the total number of families $c_{t}$ is reduced. After repeating a finite number of times the process of removing a family $c_{t}$, we obtain $F_{t}$ such that for fixed $t$ and all $i$ with $t \in B_{i}, F_{t} \cap S_{i}$ contains no contractible curves.

Our next goal is to replace $F_{t}$ by a family $F_{t}$ such that $F_{t} \cap S_{i}$ is simplified for all $i$ such that $t \in B_{i}$. The procedure for eliminating products is almost exactly the same as the procedure for eliminating contractible curves. If for some $t$ there is a product $P=W \times I / \sim$ with $\partial W \neq \varnothing, W \times 1 \subset F_{t}$ and $W \times 0 \subset S_{i}, t \in B_{i}$, then by Lemma 1.2 we can find a product satisfying $(\stackrel{\circ}{W} \times 1) \cap S_{j}=\varnothing$ for all $j$ such that $t \in B_{j}$. Such a product belongs to a continuous 1-parameter family of products $P_{t}=W_{t} \times I / \sim, t \in B_{i}$ for some $i$. As before, we find such a family with the property that, for all $t \in B_{i-1} \cap B_{i},\left(W_{t} \times 1\right) \cap S_{i-1}=\varnothing$ and that, for $t \in B_{i} \cap B_{i+1},\left(W_{t} \times 1\right) \cap S_{i+1}=\varnothing$. Then we construct a continuous family of isotopies $F_{t u}$ which eliminates the family $P_{t}$ and corresponding curves of $F_{t} \cap S_{i}$. Repeating this process, we eliminate all 1-parameter families $P_{t}$ of products.

We now have a family $F_{t}$ such that for a fixed $t, F_{t} \cap S_{i}$ is simplified for all $i$ such that $t \in B_{i}$. We must produce an isotopy $F_{t}$ so that $F_{t} \cap S$ is simplified for all $t$. This is easily achieved by modifying the family $F_{t}$ we have. For each $i$, $1 \leq i \leq n-1$, choose a point $t_{i} \in B_{i} \cap B_{i+1}$. For $t=t_{i}$, we isotope $F_{t}$ in the product between $S_{i}$ and $S_{i+1}$ so it is in Morse position with only saddle critical points at distinct levels, extending the isotopy to nearby values of $t$. This is possible by Lemma 1.1 . Finally we modify $F_{t}$ so that for a small interval $\left[v_{i}, w_{i}\right]$ containing $t_{i}, F_{t}$ is constant. Let $w_{0}=0, v_{n}=1$.

Now we are in a position to describe an isotopy $S_{t}$ of a surface isotopic to $S$. For $w_{i-1} \leq t \leq v_{i}, S_{t}=S_{i}$; for $v_{i}<t<w_{i}, S_{t}$ moves through the levels of the product between $S_{i}$ and $S_{i+1}$. Then $F_{t} \cap S_{t}$ is simplified for all $t \in I$, except at the isolated times when $S_{t}$ passes a saddle; otherwise for $t=t_{i}, F_{t}$ would have been embedded in the product between $S_{i}$ and $S_{i+1}$ with a local maximum or minimum. Isotoping $S_{t}$ back to $S$ at all times $t$ and extending the isotopy to $S_{t} \cup F_{t}$ completes the proof.

2. Proof of Theorem 1 and Theorem 3. The proofs in this section are also given only in the case $\partial M=\varnothing$.

PROOF OF THEOREM 1. Clearly we may assume that $S_{0}=S_{1}=S$ and that $F_{0}$ is isotopic to $F_{1}$, so we are in the setting of Proposition 1.2. Therefore there is an isotopy $F_{t}$ from $F_{0}$ to $F_{1}, 0 \leq t \leq 1$, such that $F_{t} \cap S$ is simplified at all times, except at the times of isolated events.

We shall prove that for all $t$ we can define a branched surface $B_{t}=F_{t} \cup S$, after suitably pinching along curves of intersection, such that

(1) $B_{t}$ is incompressible, and

(2) the sum $F_{t}+S$ in $B_{t}$ is isotopic to the sum $F_{0}+S$ in $B_{0}$. 

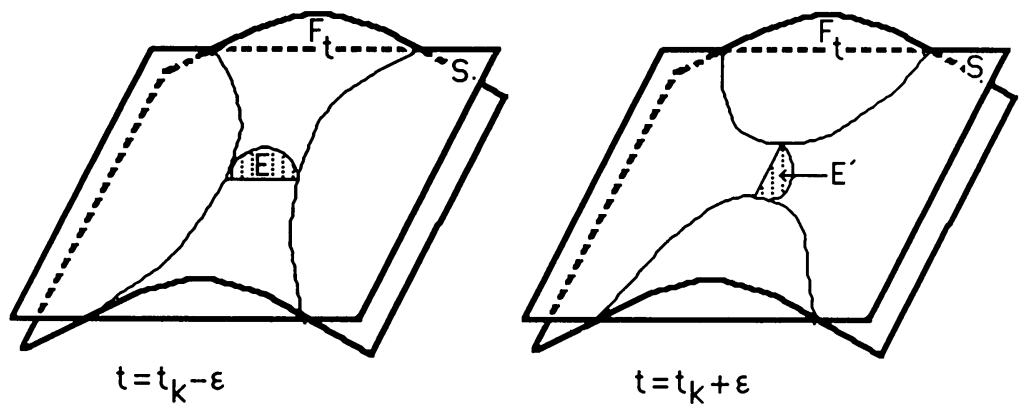

FIGURE 2.1
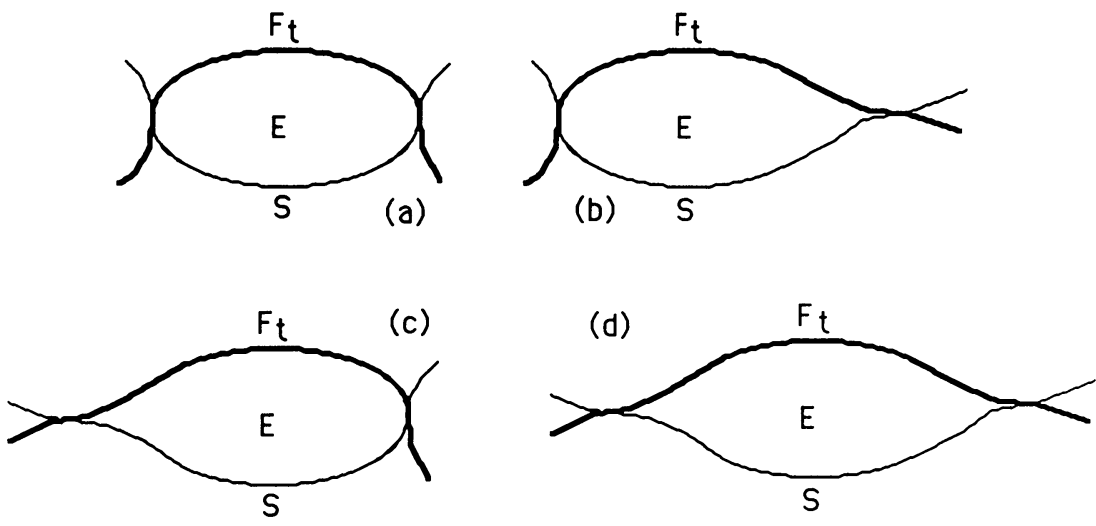

FIGURE 2.2

The curves of $F_{t} \cap S$ remain combinatorially the same as time increases, except at times of "events" of the type shown in Figure 2.1. The $k$ th event occurs at time $t=t_{k}$. As shown in the figure, there is a half-disc $E$ at time $t=t_{k}-\varepsilon$ which is replaced after the event at time $t=t_{k}+\varepsilon$ by a half-disc $E^{\prime}$.

By the hypotheses of the theorem, (1) and (2) hold when $t=0$. Suppose we have proved, inductively, that (1) and (2) hold after the $(k-1)$ th event. Then we define a branched surface structure $B_{t}$ for $F_{t} \cup S$ for $t_{k-1}<t<t_{k}$ by keeping the same sense of pinching along curves of intersection as they vary continuously. Now we consider the $k$ th event. Along each of the two (possibly identical) curves involved we have two possible pinchings. The four possibilities are shown in Figure 2.2. The two cases (b) and (c) are ruled out because they yield monogons for $B_{t}$ when $t=t_{k}-\varepsilon$. If case (a) occurs, then $E$ is a 0 -gon, which corresponds to a disc, also called $E$ say, with $E \cap \partial_{h} N\left(B_{t}\right)=\partial E$. The incompressibility of $B_{t}$, $t=t_{k}-\varepsilon$, implies that $\partial E$ bounds a disc in $\partial_{h} N\left(B_{t}\right)$, hence that the event at $t=t_{k}$ introduces a contractible curve to $S \cap F_{t}, t=t_{k}+\varepsilon$, contrary to assumption. Thus we rule out case (a). Case (d) must occur, and the same sense of pinching can be retained along the curves involved after the event to give $B_{t}$ for $t_{k}<t<t_{k+1}$.

We will show that $B_{t}$ is incompressible for $t=t_{k}+\varepsilon<t_{k+1}$, hence it is also incompressible for $t$ in the interval $\left(t_{k}, t_{k+1}\right)$. Suppose $D$ is a monogon for $B_{t} . D$ is a disc in $M-\stackrel{\circ}{N}\left(B_{t}\right)$ with $\partial D \cap \partial_{v} N\left(B_{t}\right)$ a single vertical essential arc in $\partial_{v} N\left(B_{t}\right)$. 
When we replace $B_{t}$ by $N\left(B_{t}\right), E^{\prime}$ becomes a disc $E^{\prime}$ with $\partial E^{\prime}$ intersecting $\partial_{v} N\left(B_{t}\right)$ in two vertical essential arcs. We let $D$ be transverse to the digon $E^{\prime}$. First we eliminate closed curves of $D \cap E^{\prime}$, then using half-disc surgery we can eliminate arcs of $D \cap E^{\prime}$ which have both ends on the same side of the digon $E^{\prime}$. The halfdisc we use is an innermost half-disc $H$ cut from $E^{\prime}$ by $D \cap E^{\prime}$; the surgery cuts the monogon $D$ into two pieces, a monogon and a 0 -gon. We replace $D$ by the monogon, which then intersects $E^{\prime}$ in fewer arcs than the old monogon. Thus we may assume that $D$ intersects $E^{\prime}$ only in arcs having endpoints in opposite sides of the digon. If $D \cap E^{\prime}=\varnothing$, then $D$ yields a monogon for $B_{t}, t=t_{k}-\varepsilon$, contradicting the induction hypothesis. If $D \cap E^{\prime} \neq \varnothing$, then we choose an arc $\alpha$ of $D \cap E^{\prime}$ which cuts an innermost half-disc $H$ from $D$, where $H$ is chosen so it does not contain the "vertex" ( $\operatorname{arc}$ of $\partial_{v} N\left(B_{t}\right)$ ) of the monogon. The arc $\alpha$ also cuts the digon $E^{\prime}$ in half, each half containing a vertex on its boundary. Let $K$ be one of these halves. Then $K \cup H$ yields a monogon for $B_{t}, t=t_{k}+\varepsilon$. This monogon can be isotoped to be disjoint from $E^{\prime}$, therefore it yields a monogon for $B_{t}, t=t_{k}-\varepsilon$, contrary to the induction hypothesis.

Similarly, suppose $D$ is a disc with $D \cap N\left(B_{t}\right)=\partial D \subset \partial_{h} N\left(B_{t}\right), t=t_{k}+\varepsilon$, and suppose $\partial D$ does not bound a disc in $\partial_{h} N\left(B_{t}\right)$. We call such a disc an essential 0 -gon. As before, using isotopy and half-disc surgery, we arrange that $D$ intersects $E^{\prime}$ only in arcs joining opposite sides of the digon. (Each half-disc surgery cuts $D$ into two 0 -gons, at least one of which is essential, and we replace $D$ by the new essential 0-gon which intersects $E^{\prime}$ in fewer arcs.) If $D \cap E^{\prime} \neq \varnothing$, then as before, we obtain a monogon for $B_{t}, t=t_{k}-\varepsilon$, contrary to the induction hypothesis. If $D \cap E^{\prime}=\varnothing$, then $D$ yields a corresponding 0-gon $D$ for $B_{t}, t=t_{k}-\varepsilon$. As for $E^{\prime}$ above, when we replace $B_{t}\left(t=t_{k}-\varepsilon\right)$ by $N\left(B_{t}\right), E$ becomes a disc $E$ with $\partial E$ intersecting $\partial_{v} N\left(B_{t}\right)$ in two vertical essential arcs. By the incompressibility of $B_{t}, t=t_{k}-\varepsilon, \partial D$ bounds a disc $D^{\prime}$ in $\partial_{h} N\left(B_{t}\right), t=t_{k}-\varepsilon$. Since $\partial D$ is disjoint from $E, D^{\prime}$ must be disjoint from $E$, so $D^{\prime}$ yields a corresponding disc in $\partial_{h} N\left(B_{t}\right)$, $t=t_{k}+\varepsilon$, showing that $D$ was not essential, a contradiction.

Strictly, to complete the proof that $B_{t}, t=t_{k}+\varepsilon$, is incompressible, we must also show that $\partial_{h} N\left(B_{t}\right)$ contains no sphere components, but clearly there is no possibility for the event at $t=t_{k}$ to introduce a sphere to the horizontal boundary.

It is obvious that $F_{t}+S$ for $t=t_{k}-\varepsilon$ is isotopic to $F_{t}+S$ for $t=t_{k}+\varepsilon$.

PROOF OF THEOREM 3. The proof is similar to the proof of Theorem 1. As before we assume $S_{0}=S_{1}=S$, and using Proposition 1.2, we obtain an isotopy $F_{t}$ $(0 \leq t \leq 1)$ such that $F_{t} \cap S$ is simplified at all times except at the isolated times of events of the type shown in Figure 2.1. We show by induction on events that there is a branched surface structure $B_{t}$ for $F_{t} \cup S$ such that the corresponding cut-and-paste yields a surface $G_{t}$ isotopic to $G_{0}$. Suppose we have shown that this is the case after the $(k-1)$ th event, which occurs at time $t=t_{k-1}$. Then for $t=t_{k-1}+\varepsilon<t_{k}, G_{t}$ is isotopic to $G_{0}$, hence it is incompressible.

We examine the next event at $t=t_{k}$ as before. There are four possible cases for the pinching on the two curves of $F_{t} \cap S$ involved in the event, as shown in Figure 2.2. Case (a) is ruled out because $G_{t}$ is incompressible for $t<t_{k}$ and the event would introduce a contractible curve of intersection. The argument to rule out case (c) is somewhat more complicated. Let $E$ be the monogon shown in Figure 2.2(c). We perform the cut-and-paste operation at time $t=t_{k}-\varepsilon$, and 

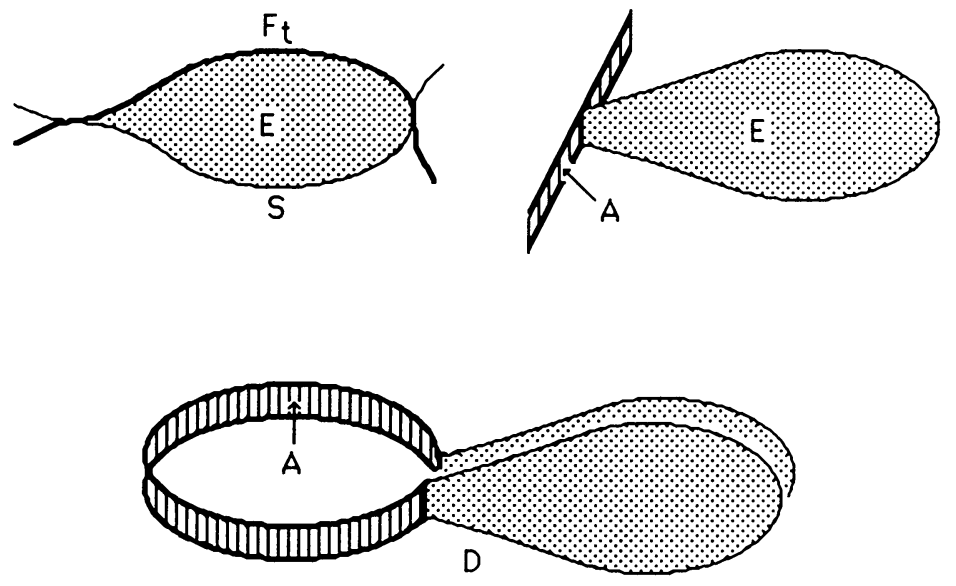

FIGURE 2.3

we replace the curve of $F_{t} \cap S$ at the vertex of the monogon $E$ by an annulus $A$ as shown in Figure 2.3. Let $N(E)=E \times[0,1]$ denote a tubular neighborhood of $E$ in the manifold obtained by cutting $M$ on $G_{t}$ and $A$. If $E_{i}=E \times i, i=0,1$, then $D=(A-N(E)) \cup E_{0} \cup E_{1}$ is a disc with $D \cap G_{t}=\partial D$ embedded in $G_{t}$ (see Figure 2.3). By the incompressibility of $G_{t}, \partial D$ bounds a disc $D^{\prime}$ in $G_{t}$, and $D \cup D^{\prime}$ bounds a ball $B^{3}$ in $M$. To determine to which side of $\partial D D^{\prime}$ lies, notice that the interior of $D^{\prime}$ is disjoint from $\partial A$, otherwise the curve of $F_{t} \cap S$ corresponding to $A$ would be contractible. For the same reason, the interior of $B^{3}$ is disjoint from $N(E)$, therefore $N(E) \cup B^{3}$ yields a solid torus with boundary $A \cup A^{\prime}$, where $A^{\prime}$ is an annulus containing $D^{\prime}$. The solid torus yields a product region between $F_{t}$ and $S, t=t_{k}-\varepsilon$. The product is of the form (annulus) $\times I$. This is a contradiction, and we have ruled out the case shown in Figure 2.2(c). Similarly we rule out (b).

The case shown in Figure 2.2(d) is the only one not ruled out. It follows that we can give a branched surface structure $B_{t}$ to $F_{t} \cup S$ for $t=t_{k}+\varepsilon$ such that the corresponding cut-and-paste yields a surface $G_{t}\left(t=t_{k}+\varepsilon\right)$ isotopic to $G_{0}$.

This completes the proof by induction.

\section{REFERENCES}

[FH] W. Floyd and A. Hatcher, The space of incompressible surfaces in a 2-bridge link complement, Trans. Amer. Math. Soc. 305 (1988), 575-599.

[FO] W. Floyd and U. Oertel, Incompressible branched surfaces via branched surfaces, Topology 23 (1984), 117-125.

[H] W. Haken, Theorie der Normalflaechen, Acta Math. 105 (1961), 245-375.

[H1] A. Hatcher, Homeomorphisms of sufficiently large $P^{2}$-irreducible 3-manifolds, Topology 15 (1976), 343-347.

[H2] - On the diffeomorphism group of $S^{1} \times S^{2}$, Proc. Amer. Math. Soc. 83 (1981), 427-430.

[JO] W. Jaco and U. Oertel, An algorithm to determine if a 3-manifold is a Haken manifold, Topology 23 (1984), 195-209.

[O1] U. Oertel, Incompressible branched surfaces, Invent. Math. 76 (1984), 385-410.

[O2] _ Measured laminations in 3-manifolds, Trans. Amer. Math. Soc. 305 (1988), 531-575.

\section{9}

Department of MAThematics, UNIVERSity of OKLAHOMA, NORMAN, OKLAHOMA 